Paidéia, 2004, 14 (28), 125 -137

\title{
REPRESENTAÇÕES SOCIAIS, RELAÇÕES INTERGRUPAIS E COGNIÇÃO SOCIAL ${ }^{1}$
}

\author{
Rosa Cabecinhas ${ }^{2}$ \\ Universidade do Minho - Portugal \\ "O pensamento é por natureza comunicativo. \\ Considerar o pensamento na cabeça de um só é \\ privá-lo de tudo o que o torna vivo e importante \\ para as pessoas".
}

Serge Moscovici

(Expresso, 25 de Janeiro de 1992)

\begin{abstract}
Resumo: Pretendemos com este artigo ilustrar as potencialidades do estudo das representações sociais em articulação com outras áreas da psicologia social, nomeadamente com as teorias das relações intergrupais e os conceitos da cognição social. Começamos por fazer referência aos principais aspectos estudados no âmbito da teoria das representações sociais: o seu conceito, a sua formação, a sua estrutura, e as funções. Seguidamente, ilustramos os pontos de convergência e de divergência com a cognição social e salientamos o papel das relações intergrupais na formação e transformação das representações sociais. Finalizamos referindo algumas das críticas de que tem sido alvo esta área de estudo no âmbito da psicologia social e de que forma estas têm vindo a ser colmatadas.
\end{abstract}

Palavras-chave: representação social; potencialidades; críticas.

\section{SOCIAL REPRESENTATIONS, INTERGRUPAL RELATIONSHIPS AND SOCIAL COGNITION}

\begin{abstract}
This article aims to illustrate the potential of the study of social representations in articulation with other areas of social psychology, namely the theories of intergroup relations and some concepts from the field of social cognition. Firstly, we review the main aspects of the theory of social representations: the concept, the process of construction of social representation, their structure and functions. Then, we discuss points of convergence and divergence in relation to the field of social cognition and emphasize the role of intergroup relations in the construction and transformation of social representations. Finally, we point out some of the criticisms that this field of study has faced within the domain of social psychology and the ways in which researchers have tried to overcome these criticisms.
\end{abstract}

Key-words:social representations; potencialities; critics.

Pretendemos com este artigo ilustrar as potencialidades do estudo das representações sociais em articulação com outras áreas da psicologia social, nomeadamente, com as teorias das relações intergrupais e alguns conceitos da cognição social.

Serge Moscovici, considerado como um dos

\footnotetext{
${ }^{1}$ Artigo recebido para publicação em 09/02/2004; aceito em 15/05/2004.

${ }^{2}$ Endereço para correspondência: Rosa Cabecinhas, Departamento de Ciências da Comunicação, Instituto de Ciências Sociais, Universidade do Minho, Campus de Gualtar, Braga, Portugal, 4710-058, E-mail: cabecinhas@ics.uminho.pt
}

"pais fundadores da psicologia social europeia" (Jesuíno, 1993, p.54), publicou em 1961 a obra La Psychanalyse, son Image et son Publique, inaugurando um novo campo na psicologia social: o estudo das representações sociais. Esta obra lançou uma problemática específica - como é que o conhecimento científico é consumido, transformado e utilizado pelo 'homem comum' (leigo) - e uma problemática mais geral - como constrói o homem a realidade. É sobre esta última problemática que nos iremos debruçar 


\section{Rosa Cabecinhas}

neste trabalho.

Moscovici conceptualiza as representações sociais como "a set of concepts, statements and explanations originating in daily life in the course of inter-individual communications” (1981, p.181). Na mesma linha de ideias, Jodelet considera-as como "une forme de connaissance, socialement élaborée et partagée, ayant une visée pratique et concourant à la construction d'une réalité commune à un ensemble social” (1989, p.36). Enquanto sistemas de interpretação, as representações sociais regulam a nossa relação com os outros e orientam o nosso comportamento. As representações intervêm ainda em processos tão variados como a difusão e a assimilação de conhecimento, a construção de identidades pessoais e sociais, o comportamento intra e intergrupal, as acções de resistência e de mudança social. Enquanto fenómenos cognitivos, as representações sociais são consideradas como o produto duma actividade de apropriação da realidade exterior e, simultaneamente, como processo de elaboração psicológica e social da realidade (Jodelet, 1989, p.36-37).

As representações sociais estão ligadas a sistemas de pensamento mais largos, ideológicos ou culturais, e a um estado de conhecimentos científicos. As instâncias institucionais, os meios de comunicação mediáticos ou informais intervêm na sua elaboração, por meio de processos de influência social. Jodelet refere ainda que as representações sociais formam sistemas e dão origem a 'teorias espontâneas', versões da realidade que incarnam em imagens cheias de significação. Através das diversas significações:

"les représentations expriment ceux (individus ou groupes) qui les forgent et donnent de l'objet qu'elles représentent une définition spécifique. Ces définitions partagées par les membres d'un même groupe construisent une vision consensuelle de la réalité pour ce groupe. Cette vision, qui peut entrer en conflit avec celle d'autres groupes, est un guide pour les actions et échanges quotidiens" (1989, p.35).

Sintetizando, as representações sociais são conceptualizadas como saber funcional ou teorias sociais práticas. Estas permitem a organização significante do real e desempenham um papel vital na comunicação: "All human interactions, whether they arise between two individuals, or between two groups, presuppose such representations. Indeed this is what characterises them” (Moscovici, 1984, p.12).

Assim, as representações sociais servem como guias da acção, uma vez que modelam e constituem os elementos do contexto no qual esta ocorre (Moscovici, 1961) e desempenham, ainda, certas funções na manutenção da identidade social e do equilíbrio sociocognitivo (Jodelet, 1989).

Na acepção de Vala (1993), afirmar que as representações são sociais envolve a utilização de três critérios: critério quantitativo - uma representação é social na medida em que é partilhada por um conjunto de indivíduos; critério genético - uma representação é social no sentido em que é colectivamente produzida (as representações sociais são o resultado da actividade cognitiva e simbólica de um grupo social); e critério funcional - as representações sociais constituem guias para a comunicação e a acção (as representações sociais são teorias sociais práticas).

Interrogado sobre o significado da passagem do conceito de representação coletiva de Durkheim para o conceito de representação social, Moscovici argumenta:

"it was the need to turn the representation into a bridge between the individual and the social worlds and to link it with a view of a changing society that led to the terminological shift [...]. It was our purpose to understand innovation rather tradition, a social life in the making rather than a preestablished one" (1988, p.219).

Na opinião de Moscovici há três formas pelas quais uma representação pode tornar-se social. As representações podem ser partilhadas por todos os membros de um grupo altamente estruturado (um partido, uma nação etc.) sem terem sido produzidas pelo grupo. Estas representações hegemónicas prevalecem implicitamente em todas as práticas simbólicas e parecem ser uniformes e coercivas. Outras representações são o produto da circulação de conhecimento e de ideias de grupos que estão em contacto mais ou menos próximo. Cada grupo cria as 
suas próprias versões e partilha-as com os outros. Estas são representações emancipadas, com certo grau de autonomia, tendo uma função complementar uma vez que resultam da partilha de um conjunto de interpretações e de símbolos. Por último, existem as representações que são geradas no decurso de um conflito ou controvérsia social e que não são partilhadas pela sociedade no conjunto. Estas representações controversas devem ser consideradas no contexto de uma oposição ou luta entre grupos (1988, pp. 221-222).

Sintetizando, as representações coletivas cedem o lugar às representações sociais porque as primeiras não têm em conta a sua diversidade de origem e a sua transformação. Segundo Moscovici (1989), a visão clássica das representações peca por as considerar como pré-estabelecidas e estáticas.

Moscovici propõe a expressão sociedade pensante para situar o estudo das representações sociais: "When we study social representations, what we are studying are human beings asking questions and looking for answers, human beings who think, not just handle information or act in a certain way" (Moscovici, 1981, p.182).

Na opinião do autor, o paradigma da sociedade pensante questiona as teorias que consideram que os nossos cérebros são 'caixas negras' que processam mecanicamente a informação em função dos condicionamentos exteriores e questiona igualmente as teorias para as quais os grupos e os indivíduos estão sempre sob o domínio das ideologias produzidas e impostas pela classe social, pelo Estado, pela Igreja ou pela Escola, e que os seus pensamentos e palavras são meros reflexos dessas ideologias. Em contrapartida, o paradigma da sociedade pensante assume que "individuals and groups are anything but passive receptors, and that they think autonomously, constantly producing and communicating representations. For them, facts, the sciences and ideologies are nothing more than 'food for thought'” (Moscovici, 1981, p.183).

A obra de Moscovici (1961) constitui um dos marcos que assinala o corte com o modelo S-O- $\mathrm{R}^{3}$ (segundo o qual as representações constituem mediações entre os estímulos e as respostas) e a emergên-

3 Abreviatura que corresponde às iniciais "Stimulus-Organism-
Response". cia do modelo O-S-O-R. Este novo modelo, atribui uma posição de primazia às representações - estas não são apenas mediações, mas fatores constituintes do estímulo e modeladores da resposta, dominando todo o processo (Markus \& Zajonc, 1985). Neste sentido, as representações são fatores produtores de realidade, determinando a forma como o indivíduo interpreta a situação e como lhe responde.

Segundo o modelo O-S-O-R tanto o estímulo como a resposta resultam da atividade construtiva do indivíduo (Moscovici, 1988, p.226), isto é, não há uma ruptura entre o universo exterior e o universo interior do indivíduo ou do grupo (Jodelet, 1989, p.39).

O estudo das representações sociais caracteriza-se por uma grande pluralidade metodológica, temática e conceptual. Vala (1993) agrupou as principais questões analisadas pela literatura nesta área nas categorias seguintes: a inscrição social e a natureza social das representações sociais; os conteúdos e a organização interna das representações sociais; a função social e a eficácia social das representações sociais; e o estatuto epistemológico das representações sociais.

Na opinião de Jodelet, as representações sociais são fenômenos complexos, permanente ativados na vida social, constituindo-se de elementos informativos, cognitivos, ideológicos e normativos (1989, p.36). Na mesma linha de idéias, Vala refere que a idéia de representação social remete para um "fenômeno psicossocial complexo, cuja riqueza torna difícil a produção de um conceito que o delimite e simultaneamente não esbata a sua multidimensionalidade" (1993, p.359).

Têm sido propostas inúmeras definições conceptuais das representações sociais, recortandoas em dimensões e aspectos específicos. Tais definições incluem na maioria dos casos conceitos de âmbito psicológico ou psicossociológico (por exemplo, atribuição, crença, atitude, esquema, opinião, etc.) e conceitos de âmbito sociológico ou antropológico tão ou mais vastos do que o próprio conceito de representação (por exemplo, ideologia, cultura, habitus, sistema de valores, etc.), relativamente aos quais o conceito de representação social "confere novas acuidades e suscita a procura de novas pontes articuladoras do velho binômio indivíduo-sociedade” 


\section{Rosa Cabecinhas}

(Vala, 1993, p.360).

Segundo Moscovici (1961), na formação das representações sociais intervêm dois processos: a objetivação e a ancoragem. Estes processos estão intrinsecamente ligados um ou outro e são modelados por fatores sociais.

A objetivação diz respeito à forma como se organizam os elementos constituintes da representação e ao percurso através do qual tais elementos adquirem materialidade, isto é, se tornam expressões de uma realidade vista como natural.

O processo de objetivação envolve três etapas. Na primeira, as informações e as crenças acerca do objeto da representação sofrem um processo de seleção e descontextualização, permitindo a formação de um todo relativamente coerente, em que apenas uma parte da informação disponível é retida. Este processo de seleção e reorganização dos elementos da representação não é neutro ou aleatório, dependendo das normas e dos valores grupais.

A segunda etapa da objetivação corresponde à organização dos elementos. Moscovici recorre aos conceitos de esquema e nó figurativo para evocar o fato dos elementos da representação estabelecerem entre si um padrão de relações estruturadas.

A última etapa da objetivação é a naturalização. Os conceitos retidos no nó figurativo e as respectivas relações constituem-se como categorias naturais, adquirindo materialidade. Isto é, os conceitos tornam-se equivalentes à realidade e o abstrato torna-se concreto através da sua expressão em imagens e metáforas.

O processo de ancoragem, por um lado, precede a objetivação e, por outro, situa-se na sua seqüência. Enquanto processo que precede a objetivação, a ancoragem refere-se ao fato de qualquer tratamento da informação exigir pontos de referência: é a partir das experiências e dos esquemas já estabelecidos que o objeto da representação é pensado. Enquanto processo que segue a objetivação, a ancoragem referese à função social das representações, nomeadamente permite compreender a forma como os elementos representados contribuem para exprimir e constituir as relações sociais (Moscovici, 1961). A ancoragem serve à instrumentalização do saber conferindo-lhe um valor funcional para a interpretação e a gestão do ambiente (Jodelet, 1989).
Vala refere que o conceito de ancoragem tem algumas afinidades com o conceito de categorização: ambos funcionam como estabilizadores do meio e como redutores de novas aprendizagens. No entanto, na opinião do autor, o processo de ancoragem é mais complexo visto que a ancoragem leva à produção de transformações nas representações já constituídas, isto é, “o processo de ancoragem é, a um tempo, um processo de redução do novo ao velho e reelaboração do velho tornando-o novo" (1993, p.363).

Os processos de objetivação e ancoragem servem para nos familiarizar com o 'novo', primeiro colocando-o no nosso quadro de referência, onde pode ser comparado e interpretado, e depois reproduzindo-o e colocando-o sob controlo (Moscovici, 1981, p.192).

As dinâmicas de objetivação e de ancoragem são aparentemente opostas:

"l'une vise à créer des vérités évidentes pour tous et indépendantes de tout déterminisme social et psychologique, l'autre désigne au contraire l'intervention de tels déterminismes dans leur genèse et transformation. Pour cette raison les études sur les représentations sociales ne peuvent pas consister seulement dans le repérage de savoirs communs, elles doivent aussi en étudier les modulations en fonction de leur imbrication spécifique dans un système de régulations symboliques" (Doise, Clémence \& Lorenzi-Cioldi, 1992, p.15).

Desde o início dos anos oitenta, numerosos estudos têm sido realizados sobre a estrutura das representações (Abric, 1984; Flament, 1989; Sá, 1996). Na opinião de Doise (1992), estes estudos permitem reduzir consideravelmente a incerteza relativa às fronteiras entre os elementos constituintes e não constituintes das representações sociais.

Flament (1989) considera que o núcleo central de uma representação corresponde a uma estrutura que dá coerência e sentido à representação. À volta do núcleo central, e organizados por este, encontramse os elementos periféricos. Estes elementos desempenham uma função importante: 
"Les schèmes périphériques assurent le fonctionnement quasi instantané de la représentation comme grille de décryptage d'une situacion: ils indiquent, de façon parfois très spécifique, ce qui est normal (et par contraste, ce qui ne l'est pas), et donc, ce qu'il faut faire comprendre, mémoriser [...]. Ces schèmes normaux permettent à la représentation de fonctionner économiquement, sans qu'il soit besoin, à chaque instant, d'analyser la situation par rapport au principe organisateur qu'est le noyau central" (Flament, 1989, p.209).

Vários estudos têm demonstrado que uma população pode ter práticas em desacordo com a representação. Na opinião de Flament (1989), estes desacordos inscrevem-se nos esquemas periféricos que se modificam protegendo, por algum tempo, o núcleo central:

\section{"Des désaccords entre réalité et représentation modifient d'abord les schèmes périphériques, puis éventuellement le noyau central, c'est-à- dire la représentation elle-même. S'il y a contradiction entre la réalité et représentation, on voit apparaître des schèmes étranges, puis une désintégration de la représentation. Si la réalité entraîne simplement une modification de l'activité des schèmes périphériques, il peut s'ensuivre une transformation progressive, mais néanmoins structurale, du noyau central" (p.218).}

A pesquisa em representações sociais apresenta um carácter fundamental e aplicado e faz apelo a metodologias variadas: experimentação no laboratório e no terreno; entrevistas; questionários; técnicas de associação livre de palavras; observação participante; análise de documentos e de discursos; etc. (Jodelet, 1989, p.5).

Doise, Clémence e Lorenzi-Cioldi (1992), numa publicação sobre as metodologias de investigação, oferecem análises bastante detalhadas sobre os laços privilegiados que existem entre os métodos de análise dos dados e os objetos teóricos no estudo das representações sociais. Como estes autores refe- rem, um problema importante nos estudos sobre as representações sociais é que a sua matéria-prima é constituída por recolhas de opinião e de atitudes individuais, sendo necessário reconstituir os princípios organizadores comuns aos conjuntos de indivíduos. Esta tarefa exige o recurso a diferentes técnicas de análises de dados.

Na opinião de Farr, um dos aspectos que caracteriza a pesquisa em representações sociais é o facto de não privilegiar nenhum método de pesquisa específico. Trata-se de uma tradição de pesquisa muito heterogénea e não prescritiva no que respeito à metodologia (1992, p.185).

No entanto, alguns autores privilegiam os estudos de terreno em detrimento dos estudos de laboratório (Farr, 1992; Moscovici, 1988). Farr (1992) argumenta:

"Les représentations sociales, par leur nature même, doivent être situées dans la culture et dans la société, non dans le laboratoire. Elles ont besoin d'être observées 'in situ', c'est-àdire sur le terrain.[...] La théorie des représentations sociales met en cause l'assertion naïve de certains expérimentateurs selon laquelle leurs laboratoires existent dans une sorte de vide culturel et temporel» (p.185).

A questão da metodologia prende-se com a questão dos níveis de análise. Doise (1982) distingue quatro níveis de análise nos trabalhos dos psicólogos sociais. No nível intra-individual estão incluídos os modelos que descrevem o modo como os indivíduos organizam a sua percepção, avaliação e comportamento em relação ao meio social em que se inserem. A interação entre o indivíduo e ambiente social é negligenciada nestes modelos - são os mecanismos que, ao nível do indivíduo, lhe permitem organizar as suas experiências, que constituem o seu objeto de análise. No nível inter-individual ou situacional encontram-se os modelos que descrevem o modo como os indivíduos interagem numa dada situação, não tomando em consideração as diferentes posições que estes possam ocupar fora dessa situação. O nível posicional integra os modelos que recorrem explicitamente às diferentes posições ou estatutos sociais que os indivíduos ocupam previamente 


\section{Rosa Cabecinhas}

a qualquer interação, para explicar as diferentes modalidades de interação, isto é, os indivíduos já não são considerados como intermutáveis. Finalmente, o nível ideológico integra os modelos que descrevem o modo como as representações e os comportamentos dos indivíduos, numa dada situação, são modelados pelos sistemas de valores, crenças e ideologias veiculadas pela sociedade.

Na opinião de Doise (1984), o estudo das representações sociais abarca diferentes níveis de análise e beneficia da sua articulação. Ora, segundo o autor, é precisamente o trabalho de articulação de níveis de análise que constitui o objeto próprio da psicologia social.

\section{Representações sociais e cognição social}

Numa revisão de literatura sobre as representações sociais, Vala (1993) sistematizou as principais diferenças entre as orientações da cognição social e as orientações na análise das representações sociais:

a) nas representações sociais, o estudo dos conteúdos e a sua articulação com contextos históricos e configurações culturais ou ideológicas é um problema nuclear, enquanto que na cognição social, os conteúdos não são, geralmente, relevantes, na medida em que se visa a elucidação de processos universais;

b) na cognição social, as questões fundamentais centram-se em torno dos processos internos através dos quais o conhecimento se forma e se transforma, enquanto que nas representações sociais, o acento tônico é colocado nos processos de interação e de influência que orientam a construção e a dinâmica do pensamento social;

c) a cognição social é orientada pela tradição molecular em psicologia (compreender o conhecimento equivale a descrever sucessivos processos e estruturas simples) enquanto que nas representações sociais o pensamento social é concebido numa perspectiva molar, articulando e envolvendo configurações de conhecimentos;

d) nas representações sociais, o plano cognitivo é articulado com os planos avaliativo e emocional, enquanto que as correntes dominantes na cognição social procuram prescindir das emoções, ainda que atualmente se façam alguns esforços no sentido da interligação das cognições, emoções e motivações (Vala, 1993, p.373).

De fato, no início dos anos noventa, o modelo dominante na cognição social considerava o indivíduo como 'avarento cognitivo' (cognitive miser). $\mathrm{O}$ ponto fulcral deste modelo é que o indivíduo é conceptualizado enquanto processador de informação de capacidade limitada, podendo lidar, num dado momento, unicamente com uma pequena quantidade de informação. Assim, o indivíduo adota estratégias para simplificar problemas complexos. Estas estratégias podem não produzir respostas normativamente corretas, mas aumentam a rapidez e a eficiência. Conseqüentemente, os erros e enviesamentos no tratamento da informação são considerados como fruto de características inerentes ao próprio sistema cognitivo, e não devidos à interferência de motivações.

No entanto, na década de noventa assistiu-se à emergência de um novo modelo na cognição social. Neste, o indivíduo é conceptualizado enquanto ‘tatico-motivado’ (motivated tactician):

" $a$ fully engaged thinker who has multiple cognitive strategies available and chooses among them based on goals, motives, and needs: sometimes the motivated tactician chooses wisely, in the interests of adaptability and accuracy, and sometimes the motivated tactician chooses defensively, in the interests of speed or self-esteem" (Fiske e Taylor, 1991, p.13).

Como Fiske (1992) refere: "After a hiatus, during which social cognition research neglected its proper atonement to social behavior, researchers again are emphasizing that thinking is for doing, that social understanding operates in the service of social interaction" (p.877; o itálico é nosso). Ora, a pesquisa elaborada no seio deste último modelo veio aproximar estas duas linhas de pesquisa.

Diversos autores consideram que uma articulação entre o paradigma da cognição social e as representações sociais poderá ser benéfica para ambas (Codol, 1984; Jahoda, 1988; Vala, 1986, 1993). Vala (1986) ilustra como ao acionar o conceito de representações sociais se consegue um olhar novo sobre 
os problemas formulados e analisados no quadro do paradigma da cognição social.

Como já referimos, a busca de articulação entre níveis de análise (Doise, 1982) constitui o ponto fulcral da matriz epistemológica que orienta o projeto da psicologia social. Vala (1993) considera particularmente frutífera a articulação entre o conceito de representação social e o conceito de esquema, e apresenta algumas semelhanças e diferenças entre estes dois conceitos.

Ambos os conceitos pressupõem que a atividade cognitiva e simbólica corresponde ao modelo O-S-O-R, isto é, ambos atribuem um papel determinante às estruturas cognitivas na produção do conhecimento social. Ambos os conceitos pressupõem um processamento da informação orientado pelas teorias (theory-driven ou top-down), por oposição a um processamento da informação orientado pelos dados (data-driven ou botton-up).

Quer os esquemas quer as representações sociais são vistos como produto de um mesmo processo básico - a categorização. E, em ambos os casos, o processo de categorização não é restringido à organização do meio, mas é também concebido como princípio organizador das relações entre os agentes sociais (Vala, 1993).

Quer os esquemas quer as representações sociais são conceptualizados como estruturas de conhecimento que guiam e facilitam o processamento da informação social. Contudo, as representações sociais, diferentemente dos esquemas, são consideradas como estando profundamente ligadas aos processos intergrupais, sendo criadas através das interações sociais.

Relativamente às diferenças: no conceito de esquema o conhecimento é conceptualizado sobretudo como resultado de um processamento de informação, onde interagem dados e teorias, enquanto que o conceito de representação social acentua as dimensões do conhecimento que resultam da aplicação das teorias, esquecendo como e de que forma essas teorias são activadas pelos dados. Assim, ao passo que os fenômenos conhecidos por erros ou enviesamentos são considerados, nas teorias sobre os esquemas, como um resultado de um processamento da informação, no quadro de análise das representações sociais esses mesmos fenômenos são resultado da uti- lização de teorias implícitas.

A pesquisa na área dos esquemas tem-se debruçado sobre a organização interna das estruturas cognitivas - este problema não tem merecido o mesmo investimento no domínio das representações sociais, com exceção dos trabalhos sobre a estrutura das representações sociais (Abric, 1984; Flament, 1989).

Esta articulação com a cognição social não deve ser confundida com uma submissão da teoria das representações sociais aos modelos da cognição social. Já referimos as revervas de Moscovici:

"Any reduction to cognitive patterns and
constructs, by eliminating the extraordinary
richness of collective thought, its rootedness
in a well-defined setting, and by flattening all
social relations into interactions between two
individuals, converts an important problem into
a mere academic exercise” (1981, p.208).

\section{Representações sociais e relações intergrupais}

A compreensão da evolução e da organização de uma representação, exige a sua integração na dinâmica social, isto é, considerá-la como determinada pela estrutura da sociedade onde se desenvolve (Moscovici, 1961). A estrutura social remete para clivagens, diferenciações e relações de dominação (Deschamps, 1982). Tais clivagens e diferenciações sociais refletem-se na construção de diferentes representações sociais de um mesmo objeto. As diferenciações no campo social registram-se pelo menos a dois níveis: o nível das condições socio-económicas; e o nível dos sistemas de orientação, desde as normas e valores mais duradouros, até atitudes e motivações específicas (Vala, 1993).

Doise (1992) considera que a significação de uma representação está sempre ancorada nas significações mais gerais que intervêm nas relações simbólicas próprias de um determinado campo social. Este autor colocou a análise das representações sociais no quadro das relações intergrupais, salientando a mútua determinação entre estes dois fenômenos.

Como Moscovici (1961) já tinha salientado, se a especificidade da situação de cada grupo social 


\section{Rosa Cabecinhas}

contribui para a especificidade das suas representações, a especificidade das representações contribui, por sua vez, para a diferenciação dos grupos sociais.

Nos seus trabalhos, Doise $(1976-84,1984)$ tem ilustrado de modo claro como a dinâmica das relações entre grupos conduz a modificações adaptativas nas representações e à atribuição ao outro grupo de características que permitem o desencadeamento de comportamentos discriminatórios e a sua justificação. Mas as representações também imprimem direção às relações intergrupais: previamente à interação, cada grupo dispõe já de um sistema de representações que lhe permite antecipar os comportamentos do outro e programar a sua própria estratégia de ação.

Na acepção de Doise, as representações assumem um lugar central nas relações intergrupais, desempenhando três tipos de funções: seleção, justificação e antecipação. A função seletiva traduz-se numa centralidade dos conteúdos relevantes para as relações intergrupais, relativamente aos conteúdos irrelevantes. A função justificativa revela-se nos conteúdos das representações que veiculam uma imagem do outro grupo que justifica um comportamento hostil em relação a ele e/ou a sua posição desfavorável no contexto da interação. Por último, a função antecipatória manifesta-se na influência que as representações exercem no próprio desenvolvimento da relação entre os grupos: as representações não se limitam a seguir o desenvolvimento das relações intergrupais, adaptando-se a ele, mas também intervêm na determinação deste desenvolvimento, antecipando-o ativamente (1976-84, p.105). A pesquisa sobre discriminação racial e étnica é bem ilustrativa destas funções das representações sociais (Cabecinhas, 2002; Vala, 1999).

Assim, por um lado, as representações estruturam-se de acordo com as estratégias grupais e, por outro, as representações servem e justificam os comportamentos grupais, isto é, as representações sociais têm uma função de justificação antecipada e/ ou retrospectiva das interações sociais (Jodelet, 1989).

Como já foi referido, a perspectiva das representações sociais enfatiza o papel ativo dos atores sociais na sua produção e transformação. É necessário, contudo, ter em conta, por um lado, a relação entre as representações sociais e as configurações culturais dominantes, e por outro, a dinâmica social no seu conjunto. A conjugação destes dois fatores ajuda a compreender as pressões para a hegemonia e a conseqüente homogeneização de certas representações sociais.

Um dos fatores responsáveis pela consensualidade alargada de algumas representações sociais, isto é, pelo seu caráter hegemônico, são os meios de comunicação social, nomeadamente a televisão. Uma representação só adquire foros de verdade e de realidade quando é partilhada - as imagens veiculadas através do ecrã têm já em si a idéia de consenso, de partilha por uma larga comunidade, o que facilita o conformismo. Por outro lado, as representações vivem de metáforas - a televisão "permite fazer corresponder a cada palavra um rosto, a cada conceito e idéia uma imagem. A expansão do audiovisual mergulhou-nos num mundo de rostos, imagens e símbolos, nos quais se inscrevem as idéias mais abstratas, conferindo-lhes a materialidade de que necessitam para viver, reproduzir-se e tornar-se realidade” (Vala, 1993, pp.379-380).

A este propósito é importante referir a afinidade entre a perspectiva prototípica da categorização social e a teoria das representações sociais. Essencialmente, o modelo prototípico postula a existência de dois princípios básicos para a formação das categorias. Primeiro, o sistema de categorias tem por objetivo fornecer a máximo de informação reduzindo o esforço cognitivo segundo o 'princípio da economia' (Rosch, 1978). Segundo, o mundo percebido não é composto por traços equiparáveis, mas por uma estrutura altamente correlacionada. O protótipo é definido como o modelo ou 'tipo ideal' que condensa o significado de uma dada categoria (Semin,1989, pp.245-246).

Como foi referido, a diferenciação das representações pode ser compreendida enquanto expressão das diferenciações no tecido social. Numa primeira perspectiva, a diferenciação das representações sociais tem sido associada a diferentes inserções dos indivíduos nos campos das estruturas socioeconômicas e socioculturais. Vala (1993) considera que esta perspectiva, por si só, não dá conta da complexidade do processo em causa. Neste quadro analítico será difícil escapar à imagem como 'homemreflexo', no contexto da qual os indivíduos são consi- 
derados como receptores passivos da ideologia dominante. Tal pressuposto vai contra o paradigma da sociedade pensante que atribui um papel ativo aos indivíduos na construção das suas representações.

Numa segunda perspectiva, parte-se do seguinte pressuposto: os indivíduos constroem representações sobre a própria estrutura social e as clivagens sociais, e é no quadro das categorias oferecidas por essas representações que se auto-posicionam e desenvolvem redes de relações, no interior das quais formam e transformam as representações sociais.

Segundo a teoria da auto-categorização do eu (Turner, Hogg, Oakes, Reicher \& Wetherell, 1987) ${ }^{4}$, um grupo existe quando os indivíduos integram na sua auto-definição a pertença a uma categoria social produzida pelo processo de categorização. Esta acepção é consonante com o conceito de identidade social proposto por Tajfel: "l'identité social d'un individu est lié à la connaissance de son appartenance à certains groupes sociaux et à la signification émotionnelle et évaluative qui résulte de cette appartenance” (1972, p.292). No entanto, é necessário ter em conta que um grupo só existe em relação a outros grupos, como salientou o autor: "les caractéristiques de son propre groupe (son statut, sa richesse ou sa pauvreté, sa couleur de peau, sa capacicité à atteindre ses buts) n'acquièrent de signification qu'en liaison avec les différences perçues avec les autres groupes ou les différences évaluatives" (1972, p.295).

Sintetizando, numa perspectiva sóciocognitiva, um grupo existe quando os indivíduos integram na sua auto-definição a pertença a uma categoria social, sendo esse processo regulado pela interdependência dos grupos sociais. Mas, na opinião de diversos autores, esta noção é demasiado restritiva

\footnotetext{
${ }^{4}$ Turner et al. (1987) definem o auto-conceito "as the set of cognitive representation of self available to a person” (p.44). O auto-conceito compreende diferentes componentes, isto é, cada pessoa possui múltiplos conceitos de si próprio. O funcionamento do auto-conceito é dependente da situação: auto-conceitos particulares tendem a ser activados e a tornar-se salientes em situações específicas. As representações cognitivas do self tomam a forma, entre outras, de auto-categorizações. Estas fazem parte de um sistema hierárquico de classificação, de acordo com o seu nível de abstracção. Na opinião de Turner et al. (1987, p.45) existem pelo menos três níveis de abstracção nas auto-categorizações: o nível supraordenado do self como ser humano; o nível intermédio referente à diferenciação endogrupo-exogrupo; e o nível subordinado correspondente às auto-categorizações pessoais baseadas na diferenciação entre o self enquanto indivíduo único e os outros membros do endogrupo.
}

(Rabbie \& Horwitz, 1988; Sherif, 1967). Para estes, um grupo só existe quando um coletivo de indivíduos se auto-percepcionam como interdependentes e possuindo objetivos comuns.

A consideração dos grupos estruturados, caracterizados pela interdependência dos seus membros e pela partilha de objetivos comuns, suscita uma terceira perspectiva de análise da formação e diferenciação das representações sociais. Bar-Tal (1989) considera que um dos fatores que melhor permitem compreender a emergência e consolidação dessas crenças é o fato de elas conferirem distintividade ao grupo, tornando-se um dos fatores constituintes das suas fronteiras. As representações partilhadas por um grupo estariam, assim, associadas à fundação do grupo e às relações intergrupais.

As relações entre as representações sociais e os processos intergrupais são bastante complexas. Alguns autores têm sublinhado a influência das posições assimétricas dos grupos, tanto nos discursos como nas identidades sociais criadas por esses grupos (Amâncio, 1994; Lorenzi-Cioldi, 1988; Tajfel, 1981-83). Embora todos os indivíduos sejam ativos na construção das suas representações, a estrutura social determina que nem todos têm igual margem de liberdade no processo de negociação das representações. Por outro lado, embora as representações estejam em permanente processo de mutação, a apropriação do 'novo' segue uma lógica de 'conservadorismo' profundamente 'sociocêntrica' (Moscovici, 1988, p.242).

\section{Reflexões sobre a "era das representações"}

Atualmente o estudo das representações sociais constitui uma tradição de pesquisa rica e diversificada e em plena evolução. A teoria das representações sociais tem hoje um alcance internacional, estando difundida muito para além das fronteiras do país natal - França. Está bem estabelecida como uma tradição de pesquisa na Europa e na América Latina e recentemente despertou o interesse dos psicólogos sociais norte-americanos.

Na opinião de diversos autores, o conceito da representação social surge como reunificador nas ciências sociais: 


\section{Rosa Cabecinhas}

"Située à l'interface du psychologique et du social, la notion a vocation pour intéresser toutes les sciences humaines. On la retrouve à l'œuvre en sociologie, anthropologie et histoire, étudiée dans ses rapports à l'idéologie, aux systèmes symboliques et aux attitudes sociales que reflètent les mentalités. [...] Cette multiplicité de relations avec des disciplines voisines confère au traitement psychosociologique de la représentation un statut transverse qui interpelle et articule divers champs de recherche, réclamant, non une juxtaposition mais une réelle coordination de leurs points de vue. Dans cette transversalité réside sans doute l'un des apports les plus prometteurs de ce domaine d'étude » (Jodelet, 1989, pp.40-41).

O conceito de representação social é actualmente utilizado por um vasto número de cientistas sociais (psicólogos, sociólogos, geógrafos, historiadores, etc.), sendo aplicado no estudo de problemas muito diversos (o ambiente, a justiça, a saúde, etc.), constituindo um campo de investigação vivo e dinâmico.

Moscovici fala mesmo na "era das representações” (1984, p.12). Com esta expressão o autor pretende veicular duas ideias fundamentais: a importância do fenómeno das representações sociais nas sociedades de hoje; e a importância do conceito de representação social no quadro das novas orientações da psicologia social.

Jesuíno (1993) refere: “No interior da PSE [psicologia social europeia] a teoria das RS [representações sociais] representa sem dúvida a tentativa mais radicalizada de rompimento com a psicologia social normal e a constituição duma disciplina alternativa, estabelecendo a ligação entre a psicologia e a sociologia, entre o indivíduo e a sociedade” (p. 58).

A pesquisa em representações sociais não está, no entanto, isenta de críticas. Estas prendem-se, sobretudo, com questões de ordem epistemológica e metodológica. Na opinião de Jesuíno, “um exame [...] dos projectos desenvolvidos [...] por estes dois autores [Moscovici e Doise] e seus associados, mostra, com efeito, que o compromisso epistemológico é precário e instável, senão mesmo insustentável” (1993, p.54).

Uma das críticas fundamentais prende-se com a definição de representação social. A este propósito Jahoda (1988) refere:

"When one looks at studies explicitly formulated in terms of social representations, there appears to be considerable arbitrariness in deciding upon appropriate units and often no attempt to justify their description as social representations.[...] The open-ended application of the term seems to provide a license to treat everything except the reified 'universe of science' as a social representation" (p.204). Por isso, este autor coloca uma importante questão: "What is not a social representation?"

Outra das críticas avançadas por Jahoda, e estreitamente relacionada com a anterior, é o fato da teoria das representações sociais estar formulada de uma forma a que não permite a sua refutação:

" $a$ scanning of the relevant literature yields no support for the claim that the theory of social representations has in any way been tested. [...] It is so loosely formulated that no part of it could readily be falsified. In comparison even psychoanalysis, to which the same reproach has been commonly addressed, constitutes a much tighter theory" (1988, p.204).

Jahoda considera ainda que, em relação aos processos vinculados às representações sociais "it would be more realistic to tie this up with the growing body of work on social cognition rather than claim the unverified existence of some special domain" (1988, p.207).

Para corrigir estas falhas, Jahoda propôs três 'remédios' para a teoria das representações sociais: formular uma definição rigorosa de representações sociais; adaptar métodos de pesquisa mais rigorosos; retornar ao quadro de referência da cognição social.

À primeira crítica de Jahoda, Moscovici (1988) replica:

"Our strategy has always been to combine the soft and hard approaches as fitted the case, 
making sure that concern for rigour did not overwhelm our heuristic concern. [...] As far as definitions go, they a moot value. Concepts have not just one but many definitions, as is true such concepts as self, schema, attitude, etc. And if there are many, then there is none. So we are left with descriptions and intuitions, some of which meet with everyone's approval and others not. While I have been reticent about defining social representations, others have done so boldly (Codol, 1984; Doise, 1984; Flament, 1989; Jodelet, 1989) and successfully” (pp.238-239).

Farr (1992) considera, no entanto, que:

"la critique de Jahoda sur la théorie est convaincante et doit être sérieusement prise en considération (Jahoda, 1988). Je pense que la réponse de Moscovici à la critique est un pas dans la bonne direction (Moscovici, 1988). On avait raison, il y a trente ans, de ne pas commencer par des définitions. Aujourd'hui, le danger est différent. Tout risque d'être considéré comme représentation sociale. Il est important de préciser ce qui n'est pas une représentation. Comment cela se rapport à d'autres termes théoriques clés dans les sciences sociales tels que attitudes, opinions, stéréotypes, idéologies, etc., doit maintenant être défini. Ceci peut désormais être fait à la lumière de la recherche empirique des trente dernières années" (p.187).

Relativamente à necessidade de uma metodologia mais rigorosa, Moscovici argumenta:

"Our reservations about rigorous methods are motivated by the need to take the growth potential of the conceptual framework into account. Being comparatively new our theory certainly still has a long way to go before being verifiable or falsifiable - on condition it remains fruitful" (1988, p.209).

Moscovici refere que o objetivo desta área de estudos é compreender "what people do in real life and in significant situations. To reach this goal, we must undoubtedly rely more on the creativity of researchers than on tried and proven procedures" (p.239). A sua reserva face a métodos de pesquisa rigorosos deve-se ao fato de querer evitar "any kind of premature exactness which [...] results in the stillbirth of capital ideas and leads to 'barren' research" (idem, p.239).

Relativamente ao retorno ao paradigma da cognição social, Moscovici rejeita com convicção a recomendação de Jahoda. A cognição social constitui o tipo de psicologia social que Moscovici não se cansa de denunciar e que, a na sua opinião, conduz inevitavelmente à subalternização da psicologia social (Jesuíno, 1993).

Moscovici (1988) argumenta:

"The body of work on social cognition studies cognition as a non-social process.[...] Social cognition pays almost no attention to the population factor and even less to the cultural factor (Pepitone, 1986). For our part, we have taken them into account and they are of great importance. The theory of social representations maintains enough flexibility to adjust to differences in groups, cultural matrices and information that circulate in a given society" (p.244).

O estudo das representações sociais ilustra uma tendência a que se assiste atualmente no seio da psicologia social de tentativa de articulação entre diversas áreas e de níveis de análise. Esta síntese, que em muitos casos se tem revelado bastante frutífera, não está, no entanto, isenta de ambigüidades.

Nas últimas décadas verificou-se um incremento considerável do número e diversidade de investigações sobre as representações sociais, tendose registrado progressos notáveis ao nível metodológico. O rompimento com a 'psicologia social normal' continua a constituir um desafio considerável e de elevado potencial.

A articulação de níveis de análise tem possibilitado a integração dos conceitos da cognição social e das terias das relações intergrupais num quadro integrado e coerente. O conceito de representação social tem permitido fazer a ponte não só entre várias 


\section{Rosa Cabecinhas}

áreas dentro da psicologia social, mas também entre as diversas ciências sociais e humanas.

\section{Referências Bibliográficas}

Abric, J. C. (1984). A theoretical and experimental approach to the study of social representations in a situation of interaction. Em R. Farr \& S. Moscovici (orgs.), Social Representations. Cambridge: University Press.

Amâncio, L. (1994). Masculino e Feminino: A Construção Social da Diferença. Porto: Afrontamento.

Cabecinhas, R. (2002). Racismo e etnicidade em Portugal: Uma análise psicossociológica da homogeneização das minorias. Dissertação de Doutoramento, Braga, Universidade do Minho.

Codol, J.P. (1984). On the system of representations in an artificial social situation. Em R. Farr \& S. Moscovici (orgs.), Social Representations. Cambridge: University Press.

Deschamps, J.C. (1982). Social identity and relations of power between groups. Em H. Tajfel (org.), Social Identity and Intergroup Relations. Cambridge: Cambridge University Press.

Doise, W. (1976-84). A Articulação Psicossociológica $e$ as Relações entre Grupos. Moraes Editores: Lisboa (tradução portuguesa de: L'Articulation Psychosociologuique et les Relations entre Groupes, Éditions A. de Boeck, Bruxelles).

Doise, W. (1982). L'Explication en Psychologie Sociale. Paris: Presses Universitaire de France.

Doise, W. (1984). Social representations, intergroup experiments and levels of analysis. Em R. Farr \& S. Moscovici (org.), Social Representations. Cambridge: University Press.

Doise, W. (1992). L’ancrage dans les études sur les représentations sociales, Bulletin de Psychologie, XLV (405), 189-195.

Doise, W., Clemence, A. \& Lorenzi-Cioldi, F. (1992). Représentations Sociales es Analyses de Données. Presses Universitaires de Grenoble.

Farr, R. (1992). La représentation sociale: la théorie et ses critiques. Bulletin de Psychologie, 405, 183-188.

Fiske, S. (1992). Thinking is for doing: portraits of social cognition from daguerreotype to laserphoto. Journal of Personality and Social Psychology, 63, 877-889.

Fiske, S.T. \& Taylor, S.E. (1991) Social Cognition. Nova Iorque: McGraw-Hill. $2^{a}$ edição.

Flament, C. (1989). Structure et dynamique des représentations sociales. Em D. Jodelet (org.), Les Représentations Sociales. Paris: Press Universitary de France.

Jahoda, G. (1988). Critical notes and reflections on 'social representations'. European Journal of Social Psychology, 18, 195-209.

Jesuíno, J.C. (1993). A psicologia social europeia. Em J. Vala \& M.B. Monteiro (Orgs.), Psicologia Social. Lisboa: Fundação Calouste Gulbenkian.

Jodelet, D. (1989). Les Représentations sociales: un domaine en expantion. Em D. Jodelet (org.), Les représentations Sociales. Paris: Press Universitary de France.

Lorenzi-Cioldi, F. (1988) Individus Dominantes et Groups Domines. Grenoble: Presses Universitaires.

Markus, H. \& Zajonc, R. B. (1985). The cognitive perspective in social psychology. Em G. Lindzey \& E. Aronson (orgs.), Handbook of Social Psychology ( $3^{\text {rd }}$ Ed.), New York: Rondom House.

Moscovici, S. (1961). La Psychanalyse, son image et son public. Paris: Press Universitary de France.

Moscovici, S. (1981). On social representations. Em J.P. Forgas (org.), Social Cognition - Perspectives on Everyday understanding. London: Academic Press.

Moscovici, S. (1984). The Phenomenon of Social Representations. Em R. Farr \& S. Moscovici (org.), Social Representations. Cambridge: University Press. 
Moscovici, S. (1988). Notes towards a description of social representations. European Journal of Social Psychology, 18, 211-250.

Moscovici, S. (1989). Des représentations collectives aux Représentations Sociales. Em D. Jodelet (org.), Les Représentations Sociales. Paris, Press Universitary de France.

Rabbie, J.M. \& Horwitz, M. (1988). Categories versus groups as explanatory concepts in intergroup relations, European Journal of Social Psychology, 18, 117-123.

Rosch, E. (1978). Human categorization. Em N. Warren (org.), Studies in Cross-Cultural Psychology, Vol.I, Londres: Academic Press.

Sá, C.P. (1996). Núcleo central das representações sociais. Petrópolis: Vozes.

Semin, G. (1989). Prototypes et représentation sociales. Em D. Jodelet (org.), Les Représentations Sociales. Paris: Press Universitary de France.

Sherif, M. (1967). Social Interaction: Process and Products. Chicago: Aldine Publ. Company.

Tajfel, H. (1972) «La catégorisation sociale». Em S. Moscovici (org.), Introduction à la Psychologie Sociale, Vol. 1. Larousse Université.

Tajfel, H. (1981-83). Grupos Humanos e Categorias Sociais, Vol. I e II, Livros Horizonte, Lda, Lisboa (tradução portuguesa de: Human Groups and Social Categories: Studies in Social Psychology, Cambridge University Press)

Turner, J. (1972). Towards a cognitive redefinition of the social group. Em H. Tajfel (ed.) Social Identity and Intergroup Relations. Cambridge: University Press.

Turner, J.C., Hogg, M.A., Oakes, P.J., Reicher, S.D. \& Wetherell, M.S. (1987). Rediscovering the Social Group: A Self-Categorization Theory. Basil Blackwell: Oxford.

Vala, J. (1986). Sobre as representações sociais - para uma epistemologia do senso comum. Cadernos de Ciências Sociais, 4, 5-30.
Vala, J. (1993). Representações sociais - para uma psicologia social do pensamento social. Em J. Vala \& M.B. Monteiro (Orgs.), Psicologia Social. Lisboa: Fundação Calouste Gulbenkian.

Vala, J. (Org.) (1999). Novos Racismos: Perspectivas Comparativas. Oeiras: Celta. 